\title{
Image Quality and Diagnostic Performance of a Digital PET Prototype in Patients with Oncologic Diseases: Initial Experience and Comparison with Analog PET
}

\author{
Nghi C. Nguyen ${ }^{1}$, Jose L. Vercher-Conejero ${ }^{1}$, Abdus Sattar ${ }^{2}$, Michael A. Miller ${ }^{3}$, Piotr J. Maniawski ${ }^{3}$, David W. Jordan ${ }^{1}$, \\ Raymond F. Muzic, Jr., ${ }^{1}$ Kuan-Hao Su${ }^{1}$, James K. O’Donnell ${ }^{1}$, and Peter F. Faulhaber ${ }^{1}$ \\ ${ }^{I}$ Department of Radiology, Case Western Reserve University/University Hospitals Case Medical Center, Cleveland, Ohio; \\ ${ }^{2}$ Epidemiology and Biostatistics, School of Medicine, Case Western Reserve University, Cleveland, Ohio; and ${ }^{3}$ Philips Healthcare, \\ Advanced Molecular Imaging, Cleveland, Ohio
}

We report our initial clinical experience for image quality and diagnostic performance of a digital PET prototype scanner with time-of-flight (DigitalTF), compared with an analog PET scanner with time-of-flight (GeminiTF PET/CT). Methods: Twenty-one oncologic patients, mean age $58 \mathrm{y}$, first underwent clinical ${ }^{18} \mathrm{~F}-\mathrm{FDG}$ PET/CT on the GeminiTF. The scanner table was then withdrawn while the patient remained on the table, and the DigitalTF was inserted between the GeminiTF PET and CT scanner. The patients were scanned for a second time using the same PET field of view with CT from the GeminiTF for attenuation correction. Two interpreters reviewed the 2 sets of PET/CT images for overall image quality, lesion conspicuity, and sharpness. They counted the number of suggestive ${ }^{18} \mathrm{~F}-$ FDG-avid lesions and provided the TNM staging for the 5 patients referred for initial staging. Standardized uptake values (SUVs) and SUV gradients as a measure of lesion sharpness were obtained. Results: The DigitalTF showed better image quality than the GeminiTF. In a side-by-side comparison using a 5-point scale, lesion conspicuity $(4.3 \pm 0.6)$, lesion sharpness $(4.3 \pm 0.6)$, and diagnostic confidence $(3.4 \pm 0.7)$ were better with DigitalTF than with GeminiTF $(P<0.01)$. In 52 representative lesions, the lesion maximum SUV was $36 \%$ higher with DigitalTF than with GeminiTF, lesion-toblood-pool SUV ratio was $59 \%$ higher, and SUV gradient was $51 \%$ higher, with good correlation between the 2 scanners. Lesions less than $1.5 \mathrm{~cm}$ showed a greater increase in SUV from GeminiTF to DigitalTF than those lesions $1.5 \mathrm{~cm}$ or greater. In 5 of 21 patients, DigitalTF showed an additional 8 suggestive lesions that were not seen using GeminiTF. In the 15 restaging patients, the true-negative rate was $100 \%$ and true-positive rate was $78 \%$ for both scanners. In the 5 patients for initial staging, DigitalTF led to upstaging in 2 patients and showed the same staging in the other 3 patients, compared with GeminiTF. Conclusion: DigitalTF provides better image quality, diagnostic confidence, and accuracy than GeminiTF. DigitalTF may be the most beneficial in detecting small tumor lesions and disease staging.

Received Sep. 10, 2014; revision accepted Jun. 29, 2015.

For correspondence or reprints contact either of the following:

Peter F. Faulhaber, Case Western Reserve University, Clinical PET, University Hospital Case Medical Center, 11100 Euclid Ave., Mailstop BSH 5056, Cleveland, OH 44106.

E-mail: Peter.Faulhaber@UHhospitals.org

Nghi C. Nguyen, Department of Radiology, 200 Lothrop St., Presbyterian

East Wing, Ste. 200, Pittsburgh, PA 15213.

Email: nguyennc@upmc.edu

Published online Jul. 9, 2015.

COPYRIGHT (c) 2015 by the Society of Nuclear Medicine and Molecular Imaging, Inc.
Key Words: digital PET; direct photon counting; ${ }^{18} \mathrm{~F}-\mathrm{FDG}$ PET; oncology

J Nucl Med 2015; 56:1378-1385

DOI: 10.2967/jnumed.114.148338

\section{$\mathbf{P}$}

ET continues to play a significant role in molecular imaging. Steady improvements in detector design and architecture as well as the implementation of time-of-flight (TOF) technology have created significant improvements in image quality and greater flexibility in reducing radiotracer dose and scanning time (1-4).

The current trend in molecular imaging places emphasis on accurate, quantitative PET imaging for improved lesion characterization and treatment monitoring (5). A new type of scintillation detector, digital photon counters (DPC), was recently developed by Philips Healthcare (6-8). The key innovation of the new digital PET system is the replacement of conventional photomultipliers with high-performance digital detectors and the implementation of singlephoton avalanche photodiodes with low-voltage complementary metal-oxide semiconductor (CMOS) logic on the same silicon substrate using CMOS process technology (6-8). This integration enables true digital photon counting without the need for noise-sensitive off-chip analog to digital conversion. In addition to digital photon counting, the detector element size matches the lutetium yttrium orthosilicate crystal size $(4 \times 4 \mathrm{~mm})$, enabling 1:1 coupling, which eliminates the need for Anger logic for crystal identification.

A PET prototype equipped with DPCs and TOF technology (DigitalTF; Philips Healthcare) was installed at the Department of Radiology, University Hospitals Case Medical Center (UHCMC), Cleveland, Ohio, in 2012 and has been operated by UHCMC and Philips Healthcare staff as part of an industry sponsored clinical trial (ClinicalTrials.gov ID, NCT01731860).

The aim of this study was to evaluate the image quality and diagnostic performance of the DigitalTF prototype in oncologic patients undergoing clinical PET/CT scanning, compared with the analog PET system with TOF, Gemini TF PET/CT (GeminiTF; Philips Healthcare).

\section{MATERIALS AND METHODS}

\section{Patient Population}

This prospective study includes patients aged $18 \mathrm{y}$ or older who were referred to the Department of Radiology for a clinical PET/CT 
examination. Patients aged 18 y or younger, or those with cognitive impairment deemed not able to give informed consent and pregnant patients were excluded from the study participation. From a pool of patients undergoing clinical ${ }^{18} \mathrm{~F}-\mathrm{FDG}$ PET/CT for initial diagnosis, staging, and follow-up of their oncologic diseases, 24 consecutive subjects were chosen to participate in this study. Of these, 3 patients were excluded from analysis because only limited imaging of the pelvis was available. Twenty-one patients were included for image review and analysis ( 9 women, 12 men; mean age $\pm \mathrm{SD}, 58 \pm 15 \mathrm{y}$ ). The study was approved by the Institutional Review Board, and all subjects signed a written consent form.

\section{Imaging Protocol}

All subjects underwent a single-injection, dual-imaging protocol including the clinical PET/CT scan (GeminiTF PET/CT) and subsequent research PET scanning (DigitalTF). For this research, we took advantage of the Gemini TF PET/CT feature that allows the PET gantry to be separated from the CT gantry by approximately 3 feet. After completion of the clinical PET/CT scan on the GeminiTF, the examination table was withdrawn, and the patient was asked to remain still on the table. The DigitalTF ring was inserted in the gap between the PET and CT gantry of the GeminiTF PET/CT scanner. Subsequently, the patients underwent an additional acquisition with DigitalTF using the same axial field of view (FOV) as GeminiTF PET/CT. In patients with a history of head and neck cancer, a clinical scan protocol usually entails scanning of the torso followed by regional scanning of the head and neck. Consequently, DigitalTF acquired only the head and neck region in these patients. The CT data of the clinical PET/CT were used for attenuation correction and anatomic correlation with DigitalTF images. Therefore, there was no additional radiation other than that already incurred during the clinical PET/CT examination.

\section{Clinical PET/CT Scanning}

The examination was performed on a GeminiTF PET/CT scanner equipped with TOF (Philips Healthcare). The patients fasted for at least $4 \mathrm{~h}$, after which they received an injection of ${ }^{18} \mathrm{~F}$-FDG. The CT consisted of a 16-slice multidetector helical CT and was obtained before the PET scan. The CT data were used for generation of the CT transmission map, image fusion, and anatomic correlation with the PET findings (GeminiTF and DigitalTF). The parameters for the CT were based on institutional guidelines: $120 \mathrm{kVp}$, pitch of 0.829 , $100 \mathrm{mAs}$ (patient weight $<68 \mathrm{~kg}$ ) or $150 \mathrm{mAs}$ (patient weight $\geq 68$ $\mathrm{kg}$ ), and 5-mm slice thickness. The scans were acquired during normal breathing. No oral or intravenous contrast was administered. The PET scanner has an active transverse FOV of $57.6 \mathrm{~cm}$. For PET scanning, the matrix size was $144 \times 144$; the voxel size was $4 \times 4 \times 4 \mathrm{~mm}$. The scan time per bed position was as follows: $1.5 \mathrm{~min} / \mathrm{bed}$, patient weight 45.4-68.0 kg; $2.0 \mathrm{~min} / \mathrm{bed}$, patient weight $68.1-90.7 \mathrm{~kg} ; 2.5 \mathrm{~min} / \mathrm{bed}$, patient weight $90.8-136.1 \mathrm{~kg}$; and $3.0 \mathrm{~min} / \mathrm{bed}$, patient weight $>136.1$ $\mathrm{kg}$. A list-mode TOF algorithm and line-of-response row-action maximum-likelihood algorithm (RAMLA) method, the so-called BLOB-OS-TF, were used for image reconstruction (2).

\section{PET Scanning for Research}

The DigitalTF prototype is an investigational device as defined per 21 CFR part 812. As a research device, the DigitalTF PET scanner is not commercially available and does not have Food and Drug Administration $510(\mathrm{k})$ clearance. The DigitalTF has the same active transverse FOV of $57.6 \mathrm{~cm}$ as but a shorter active axial FOV than the GeminiTF (16.4 vs. $18.0 \mathrm{~cm}$ ), which required more bed positions for scanning of the same body region, thus resulting in longer examination time. However, the total scanning time was the same for both scanners after adjustment of tracer decay. As with GeminiTF, the listmode TOF and BLOB-OS-TF were used for DigitalTF.

\section{Reference Standards}

Besides histopathology for the 6 patients referred for initial staging and diagnosis, 6 of 15 patients referred for restaging underwent biopsy within 3 mo before or after the imaging examination. Otherwise, the nature of the lesions (malignancy vs. benign) was based on the consensus between the 2 interpreters after review of available information in medical records.

\section{Image Review and Data Analysis}

The images were reviewed on an EBW workstation (version 4.5.3.40140; Philips Healthcare). Two experienced interpreters reviewed the 2 PET datasets separately for overall image quality using a 4-point scale (1, poor; 2 , fair; 3 , good; and 4, excellent). They subsequently compared the 2 datasets side by side for lesion conspicuity, sharpness, and diagnostic confidence on a 5-point scale (1, much worse; 2 , worse; 3 , same; 4 , better; and 5 , much better), all masked to the scanner type. The interpreters were, however, aware of the clinical indication for PET/CT. In addition, the interpreters provided the TNM staging for the 5 patients referred for initial staging, separately for GeminiTF PET/CT and DigitalTF PET/CT, interpreted $1 \mathrm{wk}$ apart. Lastly, they compared the 2 datasets side by side and counted the number of suggestive ${ }^{18} \mathrm{~F}-\mathrm{FDG}-$ avid lesions, all masked to the scanner type. The disease staging was rated according to guidelines of the American Joint Committee on Cancer.

Measurements of imaging data were obtained by 1 of the 2 interpreters. The GeminiTF PET, DigitalTF PET, and CT images were opened on the EBW workstation together, with PET/CT images being linked automatically. The primary tumor and a maximum of 3 suggestive ${ }^{18} \mathrm{~F}-\mathrm{FDG}-$ avid lesions of each tissue type (lymph node or distant metastases) with variable sizes (smallest, intermediate, largest) were included in the measurements. Whenever possible, lesions from different body regions were preferred over lesions within the same region to allow representative lesion measurements.

The shortest lesion diameter on axial CT images and standardized uptake value $(\mathrm{SUV})$, defined as (tissue concentration $[\mathrm{MBq} / \mathrm{g}] \times$ body weight $[\mathrm{g}]) /$ injected dose (MBq), were obtained. Physiologic radiotracer activity was measured in the liver, blood pool, and the area of highest myocardial uptake. A circular $1.5-\mathrm{cm}$ region of interest was placed in the mid region of the right liver lobe, in the left atrial cavity (blood pool), and in the area of highest myocardial uptake to obtain the SUVs. For all region-of-interest measurements, the same region of interest was used for both PET scanners. None of the regions of interest for the liver was placed in the area of liver metastasis. Besides mean and maximum SUV (SUV $\mathrm{Sean}_{\text {and }} \mathrm{SUV}_{\text {max }}$, respectively), we also used lesion-toblood-pool $\mathrm{SUV}_{\max }$ ratio, defined as lesion $\mathrm{SUV}_{\max } /$ blood-pool $\mathrm{SUV}_{\max }$ as a relative measure of contrast.

The measure of lesion sharpness was defined as the greatest gradient of $\mathrm{SUV}_{\text {mean }}$ between 2 adjacent pixels at the margin of an ${ }^{18} \mathrm{~F}-\mathrm{FDG}-$ avid lesion (9). A straight line was drawn through the central area of an ${ }^{18} \mathrm{~F}$ FDG-avid lesion in the axial plane, extending for approximately $1 \mathrm{~cm}$ into the background on both sides of the lesion, using the count profile tool available on the EBW workstation. The orientation of the line was not predefined but depended on the contour of the lesion. The goal was to place the line as perpendicular as possible to the lesion margin. A graph displayed the $\mathrm{SUV}_{\text {mean }}$ within each pixel along the drawn line. The greatest SUV gradients at both margins of the lesion were determined by the operator, and the averaged value was used for analysis.

Subgroup analyses were performed to assess the potential differences in lesion SUV parameters between lesions smaller than $1.5 \mathrm{~cm}$ and those $1.5 \mathrm{~cm}$ and greater. This threshold is clinically acceptable to separate smaller from larger lesions.

\section{Statistical Analysis}

Patient characteristics were assessed using measures of central tendency (mean $\pm \mathrm{SD}$; median, range of minimum to maximum) for 
continuous variables and frequencies (\%) for categoric variables. Paired Student $t$ tests, Wilcoxon tests, and median linear regressions of SUV parameters; Wilcoxon tests of image quality scores; and regression analyses were used to estimate adjusted average image quality scores and to correlate the difference in lesion $\mathrm{SUV}_{\max }$ with the time delay between the 2 PET scans. For interrater agreement and analyses of lesion conspicuity, sharpness, and diagnostic confidence, the original 5-point scores were reassigned to 3 -point scores $(1+2$ became 1,3 became 2 , and $4+5$ became 3), which would be more appropriate for the small sample size. Two-sample tests were used to determine whether the percentage changes between scan modalities for lesion subgroups were statistically significant. A $P$ value of less than 0.05 was considered statistically significant, unless specified otherwise. Bonferroni adjustments were implemented to account for multiple comparisons, with $n=3$ for lesion $\mathrm{SUV}_{\max }, \mathrm{SUV}_{\max }$ ratio, and SUV gradient. Bland-Altman analyses were used to assess the agreement between the 2 PET scans. The statistical software MedCalc 12.7.1 (MedCalc Software) and Stata 11.0 (StataCorp LP) were used.

\section{Phantom Studies}

Phantom studies were conducted to facilitate a head-to-head comparison of the DigitalTF prototype and the Gemini TF system. The methods and results of the phantom studies are provided in the supplemental materials (available at http://jnm.snmjournals.org).

\section{RESULTS}

Of the 21 patients included in this study, 15 patients were in the restaging group and 6 patients were in the initial diagnosis or staging group (Table 1). The injected ${ }^{18} \mathrm{~F}-\mathrm{FDG}$ dose was $448 \pm 67$ MBq. The uptake time for GeminiTF was $72 \pm 11 \mathrm{~min}$. The time delay between start of GeminiTF and DigitalTF was $22 \pm 12 \mathrm{~min}$. All subjects had a glucose level of less than $180 \mathrm{mg} / \mathrm{dL}$ measured within $60 \mathrm{~min}$ of ${ }^{18} \mathrm{~F}-\mathrm{FDG}$ administration, except 1 subject in whom the glucose was not obtained.

\section{Qualitative Image Quality}

In separate PET scan evaluations, the overall image quality was higher with DigitalTF (median score, 4) than with GeminiTF (median score, 3) $(P<0.01)$. The interrater agreement for overall image quality was fair, with a weighted $\kappa$ of 0.33 and $95 \%$ confidence interval (CI) of 0.1-0.6. In side-by-side comparisons, a lesion conspicuity score of $4.3 \pm 0.6$ (95\% CI, 4.4-4.8, $P<0.01)$, lesion sharpness score of $4.3 \pm 0.6(95 \% \mathrm{CI}, 4.4-4.8, P<0.01)$, and diagnostic confidence score of $3.4 \pm 0.7$ (95\% CI, 3.1-3.7, $P<$ 0.01 ) indicated that, visually, DigitalTF provided greater conspicuity, sharpness, and diagnostic confidence than GeminiTF. The interrater agreement was good for lesion conspicuity, with a weighted $\kappa$ of 0.64 (95\% CI, 0.8-1.0); very good for lesion sharpness, with a weighted $\kappa$ of 1.0 (95\% CI, 1.0-1.0); and moderate for diagnostic confidence, with a weighted $\kappa$ of 0.47 (95\% CI, 0.2-0.8).

\section{Quantitative Image Quality}

The physiologic radioactivity in the liver and blood pool, available for measurements in 18 of 21 subjects according to the imaging protocol, was slightly lower for DigitalTF than Gemini TF $(P<$ 0.05 ). Within the myocardium (measured in $13 / 21$ subjects), however, the radioactivity was markedly higher for DigitalTF than GeminiTF (Table 2). The lesion diameter was $1.2 \pm 0.9 \mathrm{~cm}$ in the 52 representative ${ }^{18} \mathrm{~F}-\mathrm{FDG}$-avid lesions. DigitalTF was $36 \%$ higher in lesion $\mathrm{SUV}_{\max }, 59 \%$ higher in $\mathrm{SUV}_{\text {max }}$ ratio, and $51 \%$ higher in SUV gradient than GeminiTF, all statistically significant based on Bonferroni adjustment (Table 2). The difference of lesion $\mathrm{SUV}_{\max }$ was not associated with the time delay between the 2 PET scans at $5 \%$ level of significance.
The SUVs for the liver, blood pool, and myocardium correlated well between the 2 modalities ( $r \geq 0.87, P<0.001$ ). The lesion SUV parameters were markedly greater for DigitalTF than GeminiTF and correlated well between the 2 scanners $(\rho \geq 0.91, P<$ $0.001)$. However, there was a systematic difference in that DigitalTF produced higher values than GeminiTF (Fig. 1).

Subgroup analyses were performed for lesions less than $1.5 \mathrm{~cm}$ (39 lesions; median size, $0.8 \mathrm{~cm}$; range, $0.4-1.3 \mathrm{~cm}$ ) and those $1.5 \mathrm{~cm}$ or greater (13 lesions; median size, $2.0 \mathrm{~cm}$; range, $1.5-5.6 \mathrm{~cm}$ ). Within these subgroups, the lesion $\mathrm{SUV}_{\max }, \mathrm{SUV}_{\max }$ ratio, and SUV gradient showed a statistically significant increase from GeminiTF to DigitalTF, as expressed in percentage (Table 3). When this percentage increase was compared between the 2 subgroups, however, they were not statistically significant although there was a tendency toward statistical significance.

There was a strong correlation between lesion $\mathrm{SUV}_{\max }$ and SUV gradient for both GeminiTF and DigitalTF (Fig. 2). In lesions less than $1.5 \mathrm{~cm}$, DigitalTF showed slightly greater enhancement in SUV gradient relative to $\mathrm{SUV}_{\max }$ than GeminiTF (slope, $5.4 x$ vs. $4.8 x$ ). These findings indicated that DigitalTF provided better lesion sharpness, particularly in smaller lesions, than GeminiTF (Fig. 2). In lesions of $1.5 \mathrm{~cm}$ or greater, both scanners showed similar correlation between lesion $\mathrm{SUV}_{\max }$ and SUV gradient (slope of $0.42 x$ with GeminiTF and $0.4 x$ with DigitalTF). Supplemental data for Figure 2 are available in the supplemental materials.

\section{Diagnostic Accuracy in Lesion Detection}

In 5 of 21 patients, DigitalTF did not miss any lesions that were seen with GeminiTF and identified an additional 8 positive lesions of more than $86{ }^{18} \mathrm{~F}-\mathrm{FDG}$-avid lesions (24\%) (Table 1). These additional lesions had a median size of $0.5 \mathrm{~cm}$ (range, $0.5-0.9$ $\mathrm{cm}$ ), with an $\mathrm{SUV}_{\max }$ of 1.0 (range, 0.8-2.2) for GeminiTF and 1.5 (range, 1.2-3.4) for DigitalTF $(P<0.005)$.

\section{Diagnostic Accuracy in Staging and Restaging}

In 15 patients presented for restaging, the true-negative rate was $100 \%$ (6 patients) for both GeminiTF and DigitalTF. Both scanners correctly identified recurrent disease in 7 of 9 patients, corresponding to a true-positive rate of $78 \%$ and false-negative rate of $22 \%$. The median size of suggestive ${ }^{18} \mathrm{~F}-\mathrm{FDG}$-avid lesions was $0.7 \mathrm{~cm}$ (range, $0.5-5 \mathrm{~cm}$ ). The corresponding $\mathrm{SUV}_{\max }$ was 3.1 (1.6-4.8) for GeminiTF and 4.5 (2.3-7.1) for DigitalTF $(P=0.02)$. False-negative cases involved 2 breast cancer patients. In a 72-y-old woman with a history of mixed lobular and ductal carcinoma, subsequent left axillary lymph node dissection revealed several metastases measuring up to $1 \mathrm{~cm}$, which were estrogen receptor- and progesterone receptor-positive. The lymph nodes were interpreted as negative with GeminiTF ( $\mathrm{SUV}_{\max }, 1.0$; blood-pool $\mathrm{SUV}_{\max }, 1.3$ ) and DigitalTF ( $\mathrm{SUV}_{\max }, 1.0$; blood-pool $\mathrm{SUV}_{\max }, 1.3$ ). In a 35-y-old woman, both PET scans were interpreted as negative for tumor. A subsequent punch biopsy of a $0.3 \times 0.3 \mathrm{~cm}$ skin lesion of the chest wall revealed adenocarcinoma with mucinous features, which was estrogen receptor-positive and progesterone receptor-negative and compatible with metastasis from the initial breast carcinoma. There was minimal ${ }^{18} \mathrm{~F}$-FDG uptake at the presumed metastatic site on both PET scans (GeminiTF: lesion $\mathrm{SUV}_{\max }, 1.25$, and blood-pool $\mathrm{SUV}_{\max }$, 1.46; DigitalTF: lesion $\mathrm{SUV}_{\max }, 1.19$, and blood-pool SUV $\mathrm{SUax}_{\max }, 1.42$ ).

Six patients presented for initial diagnosis or staging - 2 lung cancers, 1 breast cancer, 1 head and neck cancer, 1 lymphoma, 1 low-grade ganglioneuroma. The patient with ganglioneuroma 
TABLE 1

Patient Characteristics, Scanning Setup, and Imaging Diagnosis

\begin{tabular}{|c|c|c|c|c|c|c|c|c|c|}
\hline Patient no. & Age (y) & Sex & Tumor type & $\begin{array}{l}\text { Referral } \\
\text { category* }\end{array}$ & $\begin{array}{l}\text { Injected } \\
{ }^{18} \mathrm{~F}-\mathrm{FDG} \\
\text { dose } \\
\text { (MBq) }\end{array}$ & $\begin{array}{l}\text { Scan } \\
\text { range }\end{array}$ & $\begin{array}{l}\text { Time } \\
\text { delay } \\
(\min )^{\dagger}\end{array}$ & $\begin{array}{c}{ }^{18} \mathrm{~F}-\mathrm{FDG}-\text { avid } \\
\text { lesions with } \\
\text { GeminiTF }\end{array}$ & $\begin{array}{l}\text { Additional } \\
{ }^{18} \mathrm{~F}-\mathrm{FDG}- \\
\text { avid } \\
\text { lesions with } \\
\text { DigitalTF }\end{array}$ \\
\hline 1 & 52 & $\mathrm{~F}$ & Anal cancer & 2 & 433 & Torso & 20 & - & - \\
\hline 2 & 77 & $\mathrm{~F}$ & Thymic cancer & 2 & 540 & Torso & 16 & - & - \\
\hline 3 & 49 & M & $\begin{array}{l}\text { Non-Hodgkin } \\
\text { lymphoma }\end{array}$ & 2 & 385 & Torso & 29 & $\begin{array}{l}\text { Lymph } \\
\quad \text { nodes }>10\end{array}$ & - \\
\hline 4 & 61 & M & $\begin{array}{l}\text { Malignant } \\
\text { melanoma }\end{array}$ & 2 & 522 & Whole body & 55 & $\begin{array}{l}\text { Lymph } \\
\quad \text { nodes }>10\end{array}$ & $\begin{array}{l}\text { Lymph } \\
\text { node, } 1^{\ddagger}\end{array}$ \\
\hline 5 & 77 & $\mathrm{~F}$ & Multiple myeloma & 2 & 500 & Whole body & 35 & - & - \\
\hline 6 & 72 & $\mathrm{~F}$ & Breast cancer & 2 & 485 & Torso & 22 & Thyroid, 1 & - \\
\hline 7 & 51 & $\mathrm{~F}$ & Breast cancer & 2 & 503 & $\begin{array}{l}\text { Head and } \\
\text { neck }\end{array}$ & 9 & - & - \\
\hline 8 & 35 & $\mathrm{~F}$ & Breast cancer & 2 & 485 & Torso & 15 & $\begin{array}{l}\text { Lymph nodes, } \\
\text { 3/chest wall, } 1\end{array}$ & $\begin{array}{l}\text { Lymph } \\
\text { nodes, } 3^{\ddagger}\end{array}$ \\
\hline 9 & 65 & M & $\begin{array}{l}\text { Non-small cell } \\
\text { lung cancer }\end{array}$ & 2 & 414 & Torso & 17 & Bone/chest $>10$ & - \\
\hline 10 & 51 & $\mathrm{~F}$ & $\begin{array}{l}\text { Non-small cell } \\
\text { lung cancer }\end{array}$ & 2 & 400 & Torso & 16 & $\begin{array}{l}\text { Bone/chest/ } \\
\quad \text { abdomen }>10\end{array}$ & - \\
\hline 11 & 57 & M & $\begin{array}{l}\text { Non-small cell } \\
\text { lung cancer }\end{array}$ & 2 & 426 & Torso & 19 & - & - \\
\hline 12 & 81 & M & $\begin{array}{l}\text { Non-small cell } \\
\text { lung cancer }\end{array}$ & 2 & 333 & Torso & 21 & $\begin{array}{l}\text { Lung, 2/lymph } \\
\text { nodes, 2/colon, } 1\end{array}$ & Lung, $2^{\ddagger}$ \\
\hline 13 & 57 & M & $\begin{array}{l}\text { Small cell lung } \\
\text { carcinoma }\end{array}$ & 2 & 514 & Torso & 31 & $\begin{array}{l}\text { Lung, } 2 / \text { lymph } \\
\text { nodes, } 6\end{array}$ & - \\
\hline 14 & 71 & M & $\begin{array}{l}\text { Small cell lung } \\
\text { carcinoma }\end{array}$ & 2 & 440 & Torso & 18 & $\begin{array}{l}\text { Lung, 1/liver, } \\
\text { 1/lymph node, } 1\end{array}$ & - \\
\hline 15 & 30 & M & $\begin{array}{l}\text { Pleomorphic } \\
\text { sarcoma }\end{array}$ & 2 & 470 & Whole body & 42 & $\begin{array}{l}\text { Lung, } 2 / \text { lymph } \\
\text { nodes, } 2\end{array}$ & - \\
\hline 16 & 51 & M & $\begin{array}{l}\text { Non-Hodgkin } \\
\text { lymphoma }\end{array}$ & 1 & 340 & $\begin{array}{l}\text { Head and } \\
\text { neck }\end{array}$ & 9 & Lymph node, 1 & - \\
\hline 17 & 71 & M & $\begin{array}{l}\text { Non-small cell } \\
\text { lung cancer }\end{array}$ & 1 & 525 & Torso & 17 & $\begin{array}{l}\text { Primary, } 1 / \text { lymph } \\
\text { nodes, } 5\end{array}$ & - \\
\hline 18 & 47 & $\mathrm{~F}$ & Breast cancer & 1 & 400 & Torso & 16 & $\begin{array}{l}\text { Primary, 1/lymph } \\
\text { nodes, } 3\end{array}$ & $\begin{array}{l}\text { Lymph } \\
\text { node, } 1^{\Uparrow}\end{array}$ \\
\hline 19 & 32 & $\mathrm{~F}$ & Ganglioneuroma & 1 & 370 & Torso & 21 & Primary, 1 & - \\
\hline 20 & 67 & $\mathrm{~F}$ & $\begin{array}{l}\text { Head and neck } \\
\text { cancer }\end{array}$ & 1 & 374 & $\begin{array}{l}\text { Head and } \\
\text { neck }\end{array}$ & 7 & $\begin{array}{l}\text { Primary, } 1 / \text { lymph } \\
\text { node, } 1\end{array}$ & $\begin{array}{l}\text { Lymph } \\
\text { node, } 1^{\pi}\end{array}$ \\
\hline 21 & 68 & M & $\begin{array}{l}\text { Small cell lung } \\
\text { carcinoma }\end{array}$ & 1 & 555 & Torso & 24 & $\begin{array}{l}\text { Primary, } 1 / \text { chest, } \\
\text { 5/bone, } 1\end{array}$ & - \\
\hline
\end{tabular}

*2, restaging; 1, staging/initial diagnosis.

†Time delay in min between start of GeminiTF and DigitalTF.

${ }^{\ddagger}$ No difference in imaging diagnosis.

${ }^{\text {" }} \mathrm{N}$ stage is upstaged as a result of DigitalTF findings.

We did not count all ${ }^{18}$ F-FDG-avid lesions in patients with widespread disease and put label $>10$ instead.

was excluded from staging analysis because of the benign nature of the ganglioneuroma. The staging was the same with GeminiTF and DigitalTF in 3 of 5 patients $(60 \%)$ (Table 4). In the other 2 patients $(40 \%)$, however, DigitalTF led to an upstaging, compared with GeminiTF (Figs. 3 and 4). Both interpreters were concordant in all TNM staging cases. Supplemental Figures 3 and 4 are provided in the supplemental materials. For staging ( 5 patients) and restaging (15 patients) together, DigitalTF led to a change in imaging diagnosis in 2 of 20 patients (10\%), compared with GeminiTF. 
TABLE 2

Summary of Physiologic and Lesion SUV Parameters

\begin{tabular}{lccrr}
\hline \multicolumn{1}{c}{ SUV parameters } & GeminiTF & DigitalTF & $P$ & Change (\%) \\
\hline Liver SUV $_{\text {mean }}$ & $1.9 \pm 0.6$ & $1.8 \pm 0.5$ & 0.01 & -6 \\
Blood-pool SUV $_{\text {mean }}$ & $1.4 \pm 0.4$ & $1.2 \pm 0.4$ & $<0.001$ & -17 \\
Myocardium SUV $_{\max }$ & $5.6 \pm 3.0$ & $6.4 \pm 3.5$ & 0.001 & 14 \\
Lesions SUV $_{\max }$ & $5.3(1.2-19.7)$ & $7.2(0.9-24.1)$ & $<0.001^{\star}$ & 36 \\
Lesion-to-blood-pool SUV $_{\text {max }}$ ratio & $2.9(0.7-15.8)$ & $4.9(0.6-19.5)$ & $<0.001^{*}$ & 59 \\
Lesion SUV gradient & $2.0(0.3-10.1)$ & $3.0(0.6-14.2)$ & $<0.001^{*}$ & 51
\end{tabular}

*Statistically significant based on Bonferroni adjustment $(P<0.016)$.

Mean \pm SD is reported for SUV in liver, blood pool, and myocardium. For lesion SUV max $_{\text {, SUV }}$ max ratio, and SUV gradient, median, with range in parentheses, is reported for better correlation with values in Table 3 . Change (\%) indicates value change in percentage from GeminiTF to DigitalTF.

\section{DISCUSSION}

During the 2013 Radiological Society of North America annual meeting, Philips Healthcare unveiled the first commercially available Food and Drug Administration-approved PET scanner with DPC (Vereos PET/CT). In this article, we presented data pertaining to a digital PET prototype scanner that is not Food and Drug Administrationapproved but served for the clinical validation of the Vereos PET/CT. This prototype scanner has performance characteristics similar to the commercially available Vereos system (Table 5).

DPC technology, as implemented in the DigitalTF prototype, provides high detection efficiency and excellent counting rate capability. Coupled with outstanding spatial, energy, and timing resolution, this detector system promises excellent PET image quality and has the potential to improve PET quantification (6-8). Given the 1:1

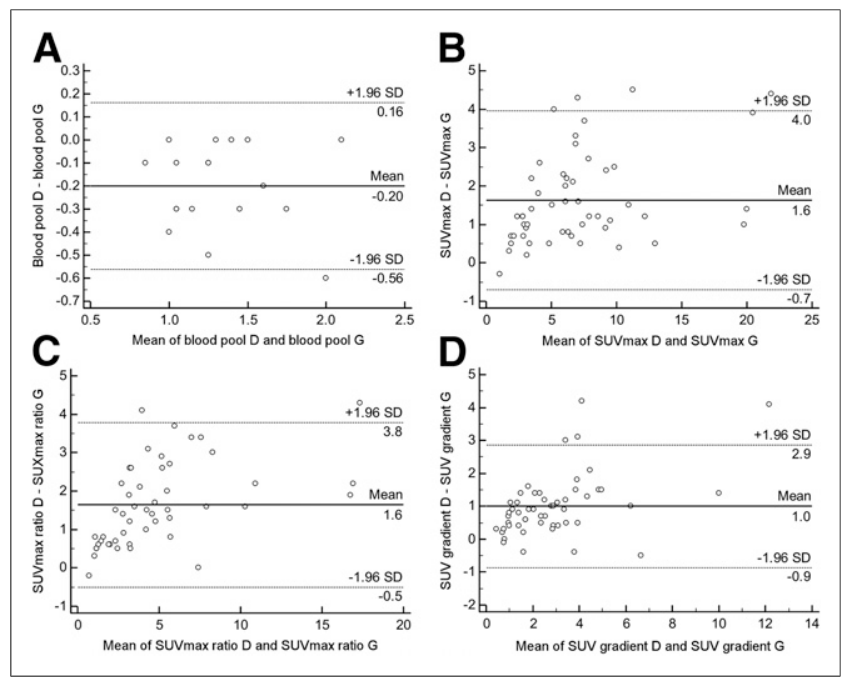

FIGURE 1. Bland-Altman plots of GeminiTF (G) and DigitalTF (D). Differences between $D$ and $G$ ( $y$-axis) are plotted against averages of $D$ and $G$ ( $x$-axis). At least $92 \%$ of difference scores fell within $95 \%$ limits of agreement. There was very good agreement between the 2 scanners for blood-pool SUV mean (A), with mean difference -0.2 , which was attributed to blood-pool clearance during time delay between PET scans. For lesion SUV max $_{\text {(B), SUV }}$ max ratio (C), and SUV gradient (D), however, there was systematic difference in that DigitalTF produced higher values than GeminiTF, with mean difference of +1.6 each for SUV $\max$ and SUV ratio and +1.0 for SUV gradient. coupling of signals, the spatial resolution is improved, and a more uniform resolution can be achieved across the detector surface. In the current study, DigitalTF provides better image quality and diagnostic performance than GeminiTF, which may have great clinical impact. To our knowledge, this is the first study to assess the image quality and diagnostic accuracy of DPC in a clinical setting. Improvements in the image quality are due to enhanced system performance in the detector, system corrections, and reconstruction parameters. The same image reconstruction algorithms were used for GeminiTF and DigitalTF, with reconstruction parameters individually optimized for both systems.

DigitalTF scored better than GeminiTF in overall image quality, lesion conspicuity, and sharpness, which is supported by the favorable interrater agreements, particularly for lesion conspicuity and sharpness. The less favorable interrater agreement for the overall image quality (weighted $\kappa, 0.33$ ) may be explained by the different appearance of background activity (noise) for DigitalTF compared with GeminiTF, which is attributed to the better spatial and TOF resolution of DigitalTF. The observed differences in SUVs in physiologic structures with low radiotracer activity (liver, blood pool) are mainly attributed to radiotracer clearance over time between the 2 PET scans (10). The $14 \%$ higher myocardial $\mathrm{SUV}_{\max }$ with DigitalTF is consistent with the fact that myocardial uptake may increase between 1 and $2 \mathrm{~h}$ after radiotracer injection (10). However, it is also possible that some of this interval increase in the myocardial uptake is partially due to improved signal detection with DigitalTF.

We found a $36 \%$ increase in lesion $\mathrm{SUV}_{\max }$ from GeminiTF to DigitalTF ( $\mathrm{SUV}_{\max }, 5.3$ vs. 7.2), which is not statistically significantly associated with the time delay. In addition, previous reports have shown less metabolic change $(<16 \%)$ despite longer time delay ( $\geq 45 \mathrm{~min}$, compared with $22 \pm 12 \mathrm{~min}$ in the current study) $(11,12)$. Thus, the observed metabolic increase in tumor lesions appears mainly attributed to the performance of DigitalTF. In addition, the Bland-Altman plots point to systematic differences between the 2 PET systems in that DigitalTF produces higher SUVrelated values than GeminiTF. Particularly, these increases are smaller for low SUVs (e.g., lesions with low ${ }^{18}$ F-FDG uptake) and larger for lesions with higher metabolic activity. These findings concur with our visual observations of increased lesion conspicuity and indicate the strengths of DigitalTF in lesion detection, compared with GeminiTF.

Along with the gain in lesion $\mathrm{SUV}_{\max }$, DigitalTF shows an improvement in lesion SUV gradient of $51 \%$. This increase in SUV gradient as a relative measure of lesion sharpness will contribute to 
TABLE 3

Subgroup Analyses of Lesion SUV Parameters Based on Size Criteria

\begin{tabular}{|c|c|c|c|c|c|}
\hline SUV parameters & GeminiTF & DigitalTF & $P$ & Change (\%) & $P$ \\
\hline Lesion SUV $\max$ & & & & & $0.078^{\star}$ \\
\hline Lesions $<1.5 \mathrm{~cm}$ & $4.6(1.2-19.7)$ & $6.3(0.9-24.1)$ & $<0.001^{\dagger}$ & 37 & \\
\hline Lesions $\geq 1.5 \mathrm{~cm}$ & $10.0(3.0-19.3)$ & $11.1(3.2-22.4)$ & $<0.001^{\dagger}$ & 11 & \\
\hline Lesion-to-blood-pool SUV $\max$ ratio & & & & & $0.02^{*}$ \\
\hline Lesions $<1.5 \mathrm{~cm}$ & $2.3(0.7-5.9)$ & $4.0(0.6-9.3)$ & $<0.001^{\dagger}$ & 72 & \\
\hline Lesions $\geq 1.5 \mathrm{~cm}$ & $7.1(2.0-15.8)$ & $8.7(2.7-19.5)$ & $<0.001^{\dagger}$ & 23 & \\
\hline Lesion SUV gradient & & & & & $0.107^{\star}$ \\
\hline Lesions $<1.5 \mathrm{~cm}$ & $1.8(0.3-10.1)$ & $2.6(0.6-14.2)$ & $<0.001^{\dagger}$ & 44 & \\
\hline Lesions $\geq 1.5 \mathrm{~cm}$ & $3.1(0.8-9.3)$ & $3.7(0.7-10.7)$ & $0.002^{\dagger}$ & 19 & \\
\hline $\begin{array}{l}{ }^{*} \text { Two-sample test-to-test proportion } \\
{ }^{\dagger} \text { Statistically significant based on Bo } \\
\text { Change (\%) indicates percentage ch }\end{array}$ & $\begin{array}{l}\text { ces between le } \\
\text { i adjustment }(P \\
\text { om GeminiTF to }\end{array}$ & $\begin{array}{l}<1.5 \mathrm{~cm} \text { and } t \\
08) . \\
\text { alTF. Values are }\end{array}$ & $\begin{array}{l}1.5 \mathrm{~cm} \text {. } \\
\text {, with rar }\end{array}$ & parentheses. & \\
\hline
\end{tabular}

a better delineation and characterization of lesions. The measured lesion sharpness takes into account the abrupt SUV change at the lesion margin and SUV change between the lesion and the background $(9,13)$. The gain in lesion sharpness seen with DigitalTF will be important when it comes to separating 2 small lesions that are located next to each other, as evidenced in Figure 3.

Our subgroup analyses stratified for lesion size again show favorable results for DigitalTF (Table 3). The percentage gain in lesion $\mathrm{SUV}_{\text {max }}, \mathrm{SUV}_{\max }$ ratio, and SUV gradient from GeminiTF to DigitalTF was at least twice as high for lesions less than $1.5 \mathrm{~cm}$ compared with those $1.5 \mathrm{~cm}$ or greater, with a tendency toward statistical significance for $\mathrm{SUV}_{\max }$ and SUV gradient. Some small lesions $(<1 \mathrm{~cm})$ showed slightly lower SUVs on DigitalTF than on GeminiTF, for which the image review did not show any plausible explanations (e.g., significant patient motion). However, it cannot be excluded that statistical noise or minor patient motion might have caused the reversed values, particularly because of the small lesion size.

Our findings are supported by performance data measured in phantoms. Spatial resolution measured using the National Electrical Manufacturers Association (NEMA) approach, including analytic reconstruction, support that the spatial resolution of the DigitalTF prototype is better than that of the GeminiTF near the center (full width at half maximum, 4.0 vs. $4.8 \mathrm{~mm})(1,14)$. Further, the percentage contrast, $\mathrm{Q}_{\mathrm{H}}$ as defined by NEMA, of the

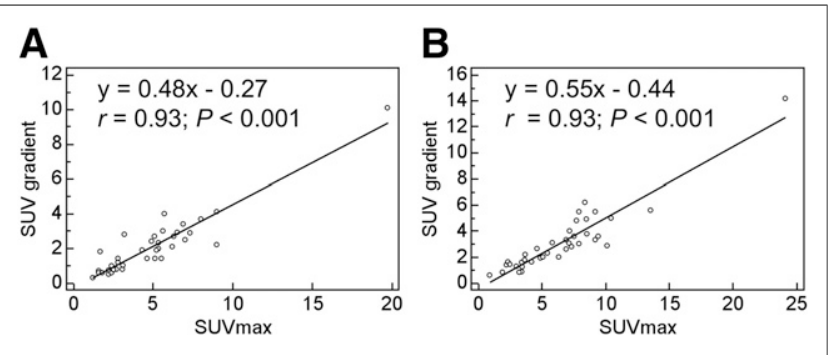

FIGURE 2. Linear regressions of SUV $V_{\max }$ and SUV gradient for lesions less than $1.5 \mathrm{~cm}$. (A) GeminiTF. (B) DigitalTF. Regression equations with correlation coefficient $r$ and $P$ value are embedded in graphs.
DigitalTF exceeds that of GeminiTF for spheres of variable sizes 10-30 mm, for durations matching clinical scans and for equal scans (Supplemental Fig. 5).

Another advantage of DPC is improved TOF timing resolution. The timing resolution was measured at $345 \mathrm{ps}$ for the Vereos scanner (Vereos PET/CT specification sheet; Philips Healthcare). The benefit of the TOF reconstruction algorithm can be realized either as noise reduction or as contrast enhancement (15). The improved TOF with the DigitalTF prototype means that individual events are distributed over a shorter distance than with GeminiTF during backprojection, leading to increased count density (higher effective sensitivity) and lower noise. The lower noise can support more aggressive postprocessing, such as the use of higher sampling (2-mm voxels as compared with the typical 4-mm voxels) and implementation of point-spread function correction.

The findings of the current study show that DigitalTF provides better diagnostic confidence than GeminiTF. The moderate interrater agreement (weighted $\kappa, 0.46$ ) may represent a bias in how much the individual interpreter expects the DigitalTF images to be better than the GeminiTF images. Nonetheless, the perceived improvement in diagnostic confidence has resulted in a detection of an additional eight ${ }^{18} \mathrm{~F}-\mathrm{FDG}$-avid lesions (measuring $<1 \mathrm{~cm}$ ) in 5 of 21 patients. Moreover, DigitalTF provides valuable information leading to upstaging in 2 of 5 patients (40\%) presented for initial staging. The current findings suggest DigitalTF would be most beneficial for detecting small lesions. As a result, DigitalTF would increase the sensitivity for ${ }^{18} \mathrm{~F}-\mathrm{FDG}-$ avid lesions and would be most helpful in the initial diagnosis and staging. Additional studies are warranted to further characterize the diagnostic performance of digital PET with DPC technology.

Although DigitalTF has shown improved lesion detection compared with GeminiTF, it failed to detect metastatic disease in 2 breast cancer patients (a $0.3-\mathrm{cm}$ skin lesion in one patient and several up to $1-\mathrm{cm}$ axillary nodes in the other patient). This is likely due to the fact the ${ }^{18}$ F-FDG PET has limited diagnostic value in the detection of skin lesions (16) and sometimes of metastatic lymph nodes from breast carcinoma (17).

Our study has some limitations. DigitalTF was performed after GeminiTF in all patients so as not to affect the imaging protocol of 
TABLE 4

Summary of PET/CT Staging

\begin{tabular}{llll}
\hline \multicolumn{1}{c}{ Primary tumor } & Staging, GeminiTF & Staging, DigitalTF & Change in staging \\
\hline Lung cancer (non-small cell) & T1NOM0 (stage I) & T1N0M0 (stage I) & No \\
\hline Lung cancer (small cell) & T3N3M1 (stage IV) & T3N3M1 (stage IV) & No \\
Breast cancer (infiltrating ductal cell) & T1N1M0 (stage II) & T1N2M0 (stage III) & Yes \\
\hline Tonsillar cancer (squamous cell) & T2N1M0 (stage II) & T2N2M0 (stage III) & Yes \\
Lymphoma of the face (large B-cell) & Stage I & Stage I & No \\
\hline
\end{tabular}

the clinical scan. Although this scan sequence might favor DigitalTF, considering that blood-pool activity decreases and tumor metabolic activity increases over time, this potential bias is probably not significant because the difference of lesion $\mathrm{SUV}_{\max }$ does not correlate well with the time delay between the 2 PET scans. Nonetheless, it is highly desirable to use a reversed scanning sequence in future studies. We acknowledge the small sample size of our study, which may be associated with large variations in the measurements. However, statistical tests account for the variability of the measures that were compared and do not invalidate a statistically significant difference. The lesion SUV

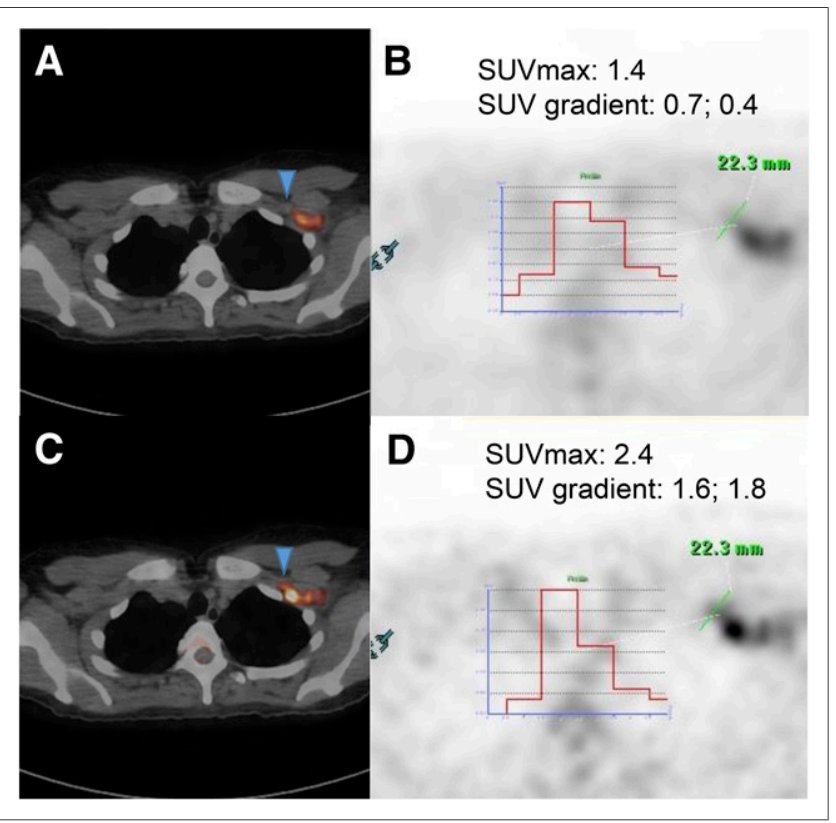

FIGURE 3. A 47-y-old woman with history of infiltrating ductal carcinoma of left breast for initial staging. Patient underwent scanning from base of skull to upper thigh on both GeminiTF and DigitalTF. GeminiTF images (upper panel) show fused axial PET/CT (A) and axial PET (B) with count profile of level III axillary lymph node (triangle), which was not diagnosed by either interpreter because of low ${ }^{18} \mathrm{~F}-\mathrm{FDG}$ avidity. Interpreters identified ${ }^{18} \mathrm{~F}-\mathrm{FDG}$-avid axillary level I and II lymph nodes, resulting in PET/CT stage II (T1N1M0). DigitalTF images (lower panel) show fused axial PET/CT (C) and axial PET (D) with count profile of same level III axillary lymph node as GeminiTF. Unlike GeminiTF, interpreters identified additional ${ }^{18} \mathrm{~F}-\mathrm{FDG}$-avid node at level III, measuring $0.9 \times 1.3$ at CT (triangle), resulting in PET/CT stage III (T1N2M0). This lesion showed greater metabolic activity and sharpness for DigitalTF (D) than GeminiTF (B). Subsequent biopsy of level I axillary node showed evidence of metastasis. gradients were manually determined on the basis of the count profile graphs, which is less accurate than computer-calculated values. However, the count profile analysis was performed by 1 person to avoid inconsistent measurements. Histopathologic verification was not possible for all suggestive lesions, but the imaging diagnosis of malignancy and the potential change in staging was highly suggestive in consensus reading. Although our quantitative results indicate a better image quality with DigitalTF than with GeminiTF, they may not reflect the actual sensitivity and specificity in clinical practice. Further studies are required to determine the diagnostic value of PET with DPC. Nonetheless, the observed improvement in image quality translates to better lesion detectability, particularly for small lesions.
A

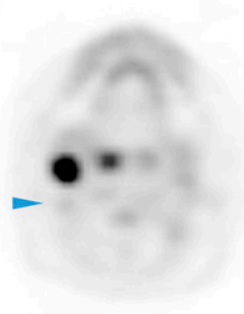

C

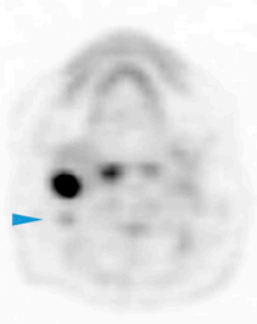

B

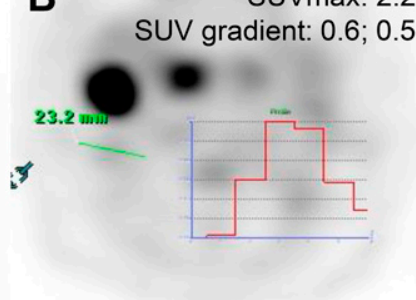

D

SUVmax: 3.4

SUV gradient: $1.4 ; 1.3$

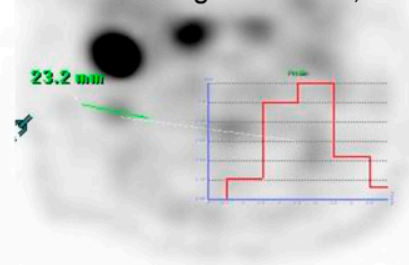

FIGURE 4. A 67-y-old woman with history of squamous cell carcinoma of right tonsil for initial staging. Patient underwent PET scanning of torso, followed by regional scanning from mid skull to upper chest for clinical care. Latter body region was scanned again with DigitalTF. GeminiTF images (upper panel) show axial PET of ${ }^{18} \mathrm{~F}-\mathrm{FDG}$-avid tonsillar primary and ${ }^{18} \mathrm{~F}-\mathrm{FDG}$-avid level IIA lymph node. Level IIB lymph node (triangle) was not diagnosed by either interpreter because of low ${ }^{18} \mathrm{~F}-\mathrm{FDG}$ uptake, for which count profile is shown in B (axial PET). PET/CT stage was II (T2N1Mx). DigitalTF images (lower panel) show axial PET and axial PET (D) with count profile of level IIB node. Unlike GeminiTF, interpreters identified this additional ${ }^{18} \mathrm{~F}-\mathrm{FDG}$-avid level IIB node, measuring $0.6 \times 0.6 \mathrm{~cm}$ at CT (triangle), resulting in PET/CT stage III (T2N2Mx). This node showed greater metabolic activity and sharpness for DigitalTF (D) than GeminiTF (B). 
TABLE 5

Data of DigitalTF Prototype, Vereos Product, and Gemini TF Product

\begin{tabular}{|c|c|c|c|}
\hline PET parameters & $\begin{array}{c}\text { DigitalTF } \\
\text { prototype } \\
\text { measurements }\end{array}$ & $\begin{array}{c}\text { Vereos product } \\
\text { data sheet } \\
452296298351 \\
\text { May } 2014\end{array}$ & $\begin{array}{c}\text { Gemini TF product } \\
\text { data sheet } \\
452296254771 \text {, } \\
\text { November } 2009\end{array}$ \\
\hline Crystal material & LYSO & LYSO & LYSO \\
\hline Detectors & $1: 1$ direct photon counting & $1: 1$ direct photon counting & Anger logic \\
\hline Crystal size (mm) & $4 \times 4 \times 22$ & $4 \times 4 \times 19$ & $4 \times 4 \times 22$ \\
\hline Transaxial FOV $(\mathrm{cm})$ & 57.6 & 57.6 (up to 67.6 ) & 57.6 (up to 67.6 ) \\
\hline Axial FOV $(\mathrm{cm})$ & 16.4 & 16.4 & 18.0 \\
\hline Energy resolution (\%) & 11.2 & 11 & 11.7 \\
\hline Timing resolution (ps) & 307 & 345 & 495 \\
\hline \multicolumn{4}{|l|}{ NEMA NU 2 spatial resolution* } \\
\hline Transverse at $1 \mathrm{~cm}$ (mm FWHM) & 3.9 & 4.1 & 4.7 \\
\hline Tangential at $10 \mathrm{~cm}(\mathrm{~mm}$ FWHM) & 4.2 & 4.5 & 5.2 \\
\hline Radial at $10 \mathrm{~cm}$ (mm FWHM) & 4.6 & 4.5 & 5.2 \\
\hline Axial at $1 \mathrm{~cm}(\mathrm{~mm}$ FWHM) & 3.8 & 4.1 & 4.7 \\
\hline Axial at $10 \mathrm{~cm}(\mathrm{~mm}$ FWHM) & 4.0 & 4.3 & 5.2 \\
\hline TOF effective sensitivity $(\mathrm{kcps} / \mathrm{MBq})^{\dagger}$ & Not measured & 22.0 & $>18.8$ \\
\hline
\end{tabular}

*Spatial resolution results are all from images reconstructed with equivalent analytic reconstruction, as specified in NU 2 standard. ${ }^{\dagger}$ Clinical sensitivity $=($ NEMA sensitivity $) \times G_{-}$tof $/ f \_D T, G \_$tof $=2 D / c \Delta t=$ TOF gain, $D=20 \mathrm{~cm} ; f_{-} D T=$ dead-time correction factor. $\mathrm{LYSO}=$ lutetium yttrium orthosilicate; FWHM $=$ full width at half maximum.

\section{CONCLUSION}

Our findings demonstrate that DigitalTF provides better image quality and diagnostic confidence as well as accuracy than GeminiTF. DigitalTF may be most beneficial in detecting and characterizing small lesions and disease staging by contributing to upstaging. Further studies are warranted to validate our findings and to assess the potential clinical impact of PET with DPC.

\section{DISCLOSURE}

The costs of publication of this article were defrayed in part by the payment of page charges. Therefore, and solely to indicate this fact, this article is hereby marked "advertisement" in accordance with 18 USC section 1734. This study was sponsored by Philips Healthcare. Drs. Faulhaber, Muzic, O'Donnell, and Sattar have a scientific cooperation contract with Philips Healthcare. Drs. Nguyen and VercherConejero were research fellows as part of collaboration between Case Western Reserve University and Philips Healthcare. Michael A. Miller and Piotr J. Maniawski are employees of Philips Healthcare. No other potential conflict of interest relevant to this article was reported.

\section{ACKNOWLEDGMENTS}

We thank Julianne Suhy, David Mercer, Sarah Ash, Sandra Dunmire, Patrick Wojtylak, and Leonard Zimmermann for their technical assistance.

\section{REFERENCES}

1. Surti S, Kuhn A, Werner ME, Perkins AE, Kolthammer J, Karp JS. Performance of Philips Gemini TF PET/CT scanner with special consideration for its time-offlight imaging capabilities. J Nucl Med. 2007;48:471-480.

2. Wang W, Hu Z, Gualtieri EE, et al. Systematic and distributed time-of-flight list mode PET reconstruction. IEEE Nucl Sci Conf R. 2006:1715-1722.
3. Hu Z, Wang W, Gualtieri EE, et al. An LOR-based fully-3D PET image reconstruction using a blob-basis function. IEEE Nucl Sci Conf R. 2007:4415-4418.

4. Surti S, Scheuermann J, El Fakhri G, et al. Impact of time-of-flight PET on whole-body oncologic studies: a human observer lesion detection and localization study. J Nucl Med. 2011;52:712-719.

5. Wahl RL. 2013 SNMMI highlights lecture: oncology. J Nucl Med. 2013;54:11N-22N.

6. Frach T, Prescher G, Degenhardt C, de Gruyter R, Schmitz A, Ballizany R. The digital silicon photomultiplier: principle of operation and intrinsic detector performance. IEEE Nucl Sci Conf R. 2009:1959-1965.

7. Degenhardt C, Prescher G, Frach T, et al. The digital silicon photomultiplier: a novel sensor for the detection of scintillation light. IEEE Nucl Sci Conf R. 2009:2383-2386.

8. Degenhardt C, Rodrigues P, Trindade A, et al. Performance evaluation of a prototype positron emission tomography scanner using digital photon counters (DPC). IEEE Nucl Sci Symp Med Imaging Conf Rec. 2012:2820-2824.

9. Geets X, Lee JA, Bol A, Lonneux M, Gregoire V. A gradient-based method for segmenting FDG-PET images: methodology and validation. Eur J Nucl Med Mol Imaging. 2007;34:1427-1438.

10. Cheng G, Alavi A, Lim E, Werner TJ, Del Bello CV, Akers SR. Dynamic changes of FDG uptake and clearance in normal tissues. Mol Imaging Biol. 2013;15:345-352.

11. Cheng G, Torigian DA, Zhuang H, Alavi A. When should we recommend use of dual time-point and delayed time-point imaging techniques in FDG PET? Eur $J$ Nucl Med Mol Imaging. 2013;40:779-787.

12. Kumar R, Loving VA, Chauhan A, Zhuang H, Mitchell S, Alavi A. Potential of dual-time-point imaging to improve breast cancer diagnosis with ${ }^{18} \mathrm{~F}$-FDG PET. J Nucl Med. 2005;46:1819-1824.

13. Xu J, Napel S, Greenspan H, Beaulieu CF, Agrawal N, Rubin D. Quantifying the margin sharpness of lesions on radiological images for content-based image retrieval. Med Phys. 2012;39:5405-5418.

14. Miller MA, Jordan D, Laurence T, et al. Initial characterization of a prototype digital photon counting PET system [abstract]. J Nucl Med. 2014;55(suppl 1):658.

15. Karp JS, Surti S, Daube-Witherspoon ME, Muehllehner G. Benefit of time-offlight in PET: experimental and clinical results. J Nucl Med. 2008;49:462-470.

16. Böni R, Böni RA, Steinert H, et al. Staging of metastatic melanoma by wholebody positron emission tomography using 2-fluorine-18-fluoro-2-deoxy-D-glucose. Br J Dermatol. 1995;132:556-562.

17. Filippi V, Malamitsi J, Vlachou F, et al. The impact of FDG-PET/CT on the management of breast cancer patients with elevated tumor markers and negative or equivocal conventional imaging modalities. Nucl Med Commun. 2011;32:85-90. 